\title{
Effects of the Simplification in the Geometry of a Cyclone Test Facility on the Swirling Flow: a CFD Study
}

\author{
Evandro Balestrin ${ }^{\mathrm{a}}$, Rodrigo K. Decker ${ }^{\mathrm{b}}$, Jaci C. S. C. Bastos ${ }^{\mathrm{b}}$ and Henry F. Meier ${ }^{\mathrm{b}}$
}

${ }^{a}$ Federal University of Santa Catarina, Department of Chemical and Food Engineering, 88040-900, Florianópolis-SC, Brazil
${ }^{b}$ University of Blumenau, Department of Chemical Engineering, São Paulo St., 3250, Room I-204, 89030-000, Blumenau-SC, Brazil

ARTICLE INFO

\section{Keywords:}

Computational,

fluidynamic,

turbulence,

Reynolds Stress

Model, cyclone,

swirling.

\begin{abstract}
A study about the influence of geometry simplification on the swirling flow of a cyclone was performed by comparing CFD results with experimental data. The numerical results were obtained by solving the mass and momentum equations with the Reynolds Stress Model (RSM) for the turbulence closure. On other hand, the experimental data were obtained in the literature, the authors used the Particle Image Velocimetry (PIV) technique to measure the velocity fields and a differential manometer to measure the pressure drop. In this work, different test facility geometries configurations were simulated: the complete test facility; and others with some simplifications downstream and upstream of the cyclone. The boundary condition for both numerical and experimental analysis was performed with inlet velocity in 10.5 and $12.25 \mathrm{~m} / \mathrm{s}$. Results showed a decrease in the gas vortex velocity in the cyclone center when simplifications in the test facility geometry are made. These bring significant consequences in the performance parameters, like almost $20 \%$ in the pressure drop values.
\end{abstract}

other words, without considering the apparatus before and after or even the real shape of the devices under study. These basic geometries may result in simulation time gain and computational resource-saving, such as presented by Vatankhah et al. ${ }^{[11]}$. On other hand, this process should be very carefully conducted to not lose essential information regarding the physical phenomena. Taghinia et al. ${ }^{[12]}$ showed that simplification of body shape on the prediction of the flow field around a dummy could reach $10 \%$.

Regarding separator cyclones, in Schmidt et al. ${ }^{[13]}$ it is possible to analyze the difference in the flow results between the real cyclone geometry and those with boundary modifications. It was found that only a limited amount of literature exists about the effects of the geometry simplifications in cyclones test facilities to performed CFD studies. So, this study presents the effects of the simplifications in the geometry of a cyclone test facility on the swirling flow and pressure drop by comparing experimental data and numerical results.

\section{Material and Methods}

This section is divide into two parts, the former regarding the material and methods used by Balestrin et al. ${ }^{[10]}$ to obtain the experimental data, and the latter those used in the numerical simulations to reach the aim of this study.

\subsection{Overview of experiments}

The experiments were performed in the test facility, as illustrated in Figure 1. The experimental unit operates with an exhauster (10) at the end of the line, which produces a negative pressure that transports the gas. Flow beginning in the inlet duct (01) which passes into the cyclone (06) toward the bag filter (09) until exit. 


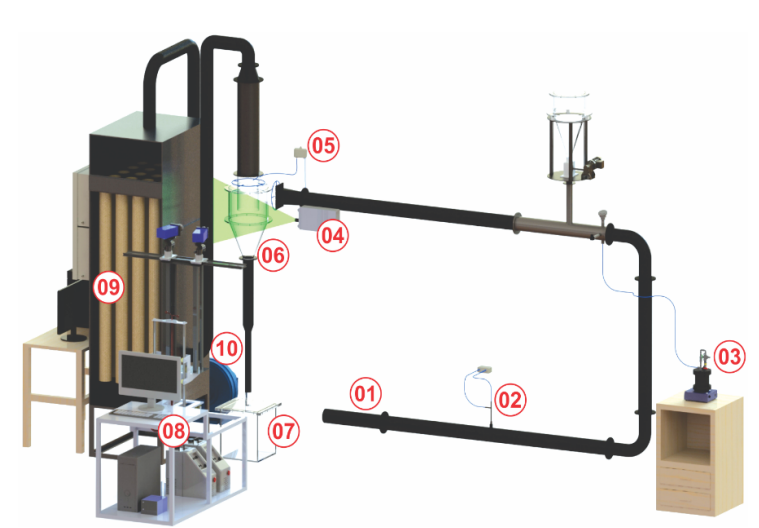

Figure 1: Test facility and measurement system applied in physical experiments. 01 Inlet duct; 02 Pitot tube; 03 Tracer feeder; 04 Laser; 05 Pressure probe; 06 Cyclone; 07 Solid collector; 08 PIV; 09 Bag filter; 10 Exhauster.

Macroscopic and microscopic physical quantities were obtained from the gas phase in the test facility, such as pressure drop and velocity fields. Experiments were performed at two operation conditions, a duct inlet velocity of 10.5 and $12.25 \mathrm{~m} / \mathrm{s}$. Pressure loss was obtained by the difference of inlet and outlet pressure measure points using a pressure probe (05). Velocity fields were achieved using a particle image velocity system (PIV). The PIV system (08) is composed of a computer, two cameras, and a laser (04). A particle tracer with a Sauter diameter of about $4 \mu \mathrm{m}$ and density of $1400 \mathrm{~kg} / \mathrm{m}^{3}$ was fed in the flow to perform the experiments.

The physical experimental data was used to compare with the numerical simulation results, and therefore, present the loss of information due to the geometry simplification performed in the test facility.

\subsection{Numerical simulations}

Numerical simulations were developed in FLUENT 14.0. The simulations were conducted in transient-state with time step and convergence criteria of $10^{-4}$. The SIMLPEC algorithm was chosen for pressure-velocity coupling. The second-order upwind discretization scheme was used except for pressure where PRESTO was selected. For boundary conditions, inlet velocity, pressure outlet and a stationary wall were set.

Four different geometry configurations of the test facility were simulated to demonstrate the loss of information in pressure drop, axial velocity and tangential velocity when confronted with experimental data. Figure 2 showed the distinct cases simulated and the dimensions in millimeters of the cyclone, inlet and outlet

\subsection{Mathematical Modelling}

To predict the cyclone swirling gas flow, Navier-Stokes equations (mass and momentum) were applied, with the Reynolds Average Navier-Stokes (RANS) equations approach and under the Eulerian focus.

\section{Mass equation}

$$
\frac{\partial}{\partial t}\left(\rho_{g}\right)+\nabla \cdot\left(\rho_{g} v_{g}\right)=0
$$

Momentum equation

$\frac{\partial}{\partial t}\left(\rho_{g} v_{g}\right)+\nabla \cdot\left(\rho_{g} v_{g} v_{g}\right)=-\nabla \cdot\left(T_{g}^{e f f}\right)+\rho_{g} g-\nabla p$

The subscript " $g$ " indicates that these equations are valid to the gas phase. Gas is the main phase in order to characterize the flow, therefore the focus of this study. Term $\left(T_{g}^{e f f}\right)$ is the effective stress, which expresses the sum of the turbulent Reynolds stress term $\left(T_{g}^{t}\right)$ and the term of the molecular tensor $\left(T_{g}\right)$, such as follow: $T_{g}^{e f f}=T_{g}^{t}+T_{g}$. extensions that constitute the experimental unit. The differences of each case are discussed below:

- $\quad$ Figure 2a (Case 0), this case has got the same geometry configuration of the test facility, but ending before entering the bag filter;

- Figure 2b (Case 1), this case is similar to Case 0 but without the inlet duct and the tube after the reduction section located in the gas outlet tube;

- $\quad$ Figure 2c (Case 2), this case is similar to Case 1 but without the reduction section in the gas outlet pipe;

- Figure 2d (Case 3), this case is similar to Case 2 but without the extension of the cyclone gas outlet duct.

A mesh with about five million hexaedral elements was used for Case 0 . The choice of the mesh was based on a grid convergence index (GCl) study performed by Balestrin et al. ${ }^{[10]}$. The mesh of Case 1, Case 2 and Case 3 have the same characteristics as the Case 0 mesh.

All the cases were simulated at the operational conditions used in the physical experiments, gas inlet velocity in 10.5 and $12.25 \mathrm{~m} / \mathrm{s}$. Cases 1,2 and 3 , compared to the Case 0 and the experimental data show the loss of information in the studied parameters incurred after the simplifications in the CFD geometry.

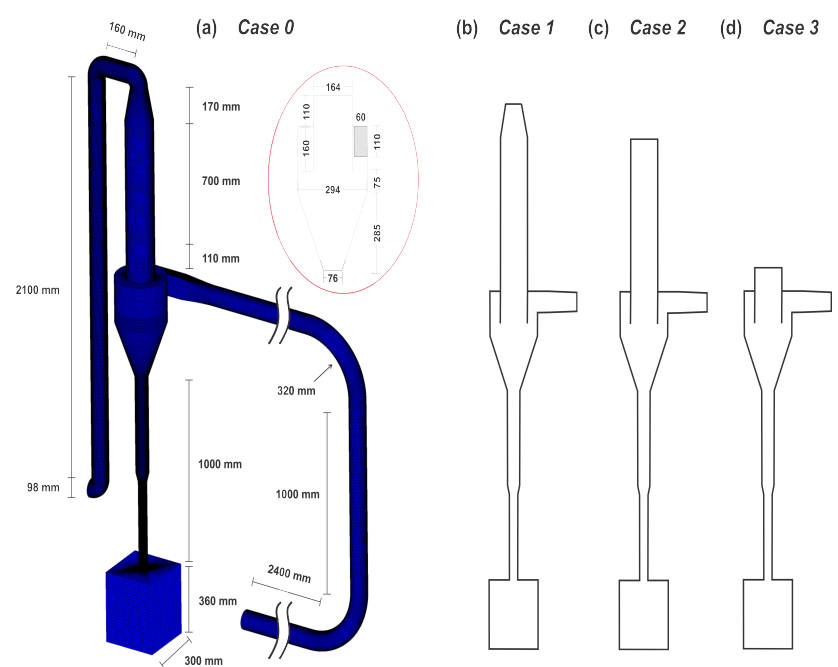

Figure 2: Four distinct geometries used in the numerical simulations and their main dimension

The nature of the cyclone turbulence is highly anisotropic due to its spiraled motion. So, the anisotropic Reynolds Stress Model (RSM) was used to better represent the swirling flow. This model requires a transport equation for each Reynolds stress component and to the dissipation rate, totaling seven more equations to close the turbulence problem. Eq (4) expresses the Reynolds transport equation.

$\frac{\partial}{\partial \mathrm{t}}\left(T_{g}^{t}\right)+\nabla \cdot \mathrm{v}_{\mathrm{g}}\left(T_{g}^{t}\right)=\mathrm{D}_{\mathrm{T}}+\mathrm{D}_{\mathrm{M}}+\Upsilon+\Pi-\frac{2}{3} \delta \varepsilon_{\mathrm{g}}$.

Turbulence diffusion $\left(D_{T}\right)$, which is presented by Lien and Leschziner ${ }^{[14]}$ was modeled as:

$\mathrm{D}_{\mathrm{T}}=\nabla \cdot\left(\frac{v_{g}^{t}}{\sigma_{k}} \nabla \mathrm{T}_{g}^{t}\right)$.

Molecular diffusion $\left(\mathrm{D}_{\mathrm{M}}\right)$ is expressed by:

$\mathrm{D}_{\mathrm{M}}=\nabla \cdot\left(v_{g} \nabla \mathrm{T}_{g}^{t}\right)$.

Production rate $(Y)$ is represented by Eq (7).

$\Upsilon=-\left[\mathrm{T}_{g}^{t} \cdot\left(\nabla \mathrm{v}_{\mathrm{g}}\right)^{\mathrm{T}}+\mathrm{T}_{g}^{t} \cdot\left(\nabla \mathrm{v}_{\mathrm{g}}\right)\right]$. 
The strain deformation term (П) can be expressed in different shapes, such as linear modeling, quadratic modeling and others. Due to a preliminary test by Balestrin et al. ${ }^{[10]}$, that showed no significant difference between quadratic and linear modeling over the evaluated parameters and the easy convergence of the linear modeling, the latter was applied. Fu et al. ${ }^{[15]}$ and Gibson and Lauder ${ }^{[16]}$ present more information about RSM modeling.

The conservation equation needed for the dissipation rate is shown in Eq (8).

$$
\begin{aligned}
\frac{\partial}{\partial t}\left(\rho_{g} \varepsilon_{g}\right)+\nabla \cdot\left(\rho_{g} \mathrm{v}_{\mathrm{g}} \varepsilon_{\mathrm{g}}\right) & \\
= & \nabla \cdot\left[\left(\mu-\frac{\mu_{g}^{t}}{\sigma_{\varepsilon}}\right) \nabla \varepsilon_{g}\right] \\
& +\frac{\varepsilon_{g}}{\mathrm{k}_{g}} \rho_{g} \mathrm{C}_{\varepsilon 1} \Upsilon^{*}-\frac{\varepsilon_{g}{ }^{2}}{\mathrm{k}_{g}} \rho_{g} \mathrm{C}_{\varepsilon 2} .
\end{aligned}
$$

Where the turbulent viscosity $\left(\mu_{g}^{t}\right)$ is represented as:

$$
\mu_{g}^{t}=\mathrm{C}_{\mu} \rho_{g} \frac{k_{\mathrm{g}}{ }^{2}}{\varepsilon_{\mathrm{g}}} \text {. }
$$

The model's constant values applied were the default values used in the FLUENT 14.0 code. For a better prediction of the experimental data, the parameters can be adjusted according to a sensitivity study

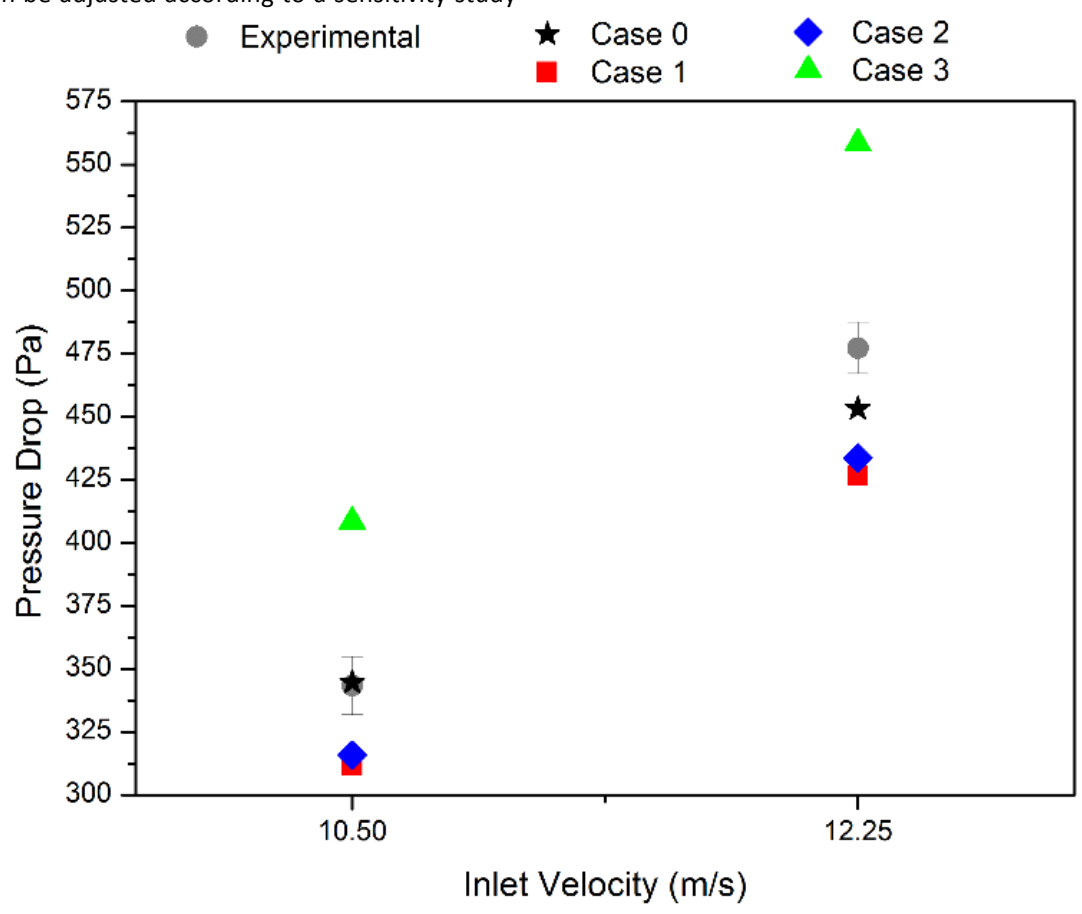

Figure 3: Pressure drop comparison for all studies cases, inlet velocity in 10.5 and $12.25 \mathrm{~m} / \mathrm{s}$.

Case 0
Case 1 $\quad \rightarrow \begin{aligned} & \text { Case } 2 \\ & \text { Case } 3\end{aligned}$

$\Delta$ such as conducted by Balestrin et al. ${ }^{[17]}$, or by an optimization study. The standard wall function was used as the near-wall treatment.

\section{Results and Discussions}

To illustrate the information loss in the cyclone flow due to geometry simplifications, pressure drop, axial velocity and tangential velocity were analyzed.

\subsection{Pressure drop results}

Figure 3 presents the pressure drop comparison of all proposed numerical case results with the experimental data. The bar in the experimental value of pressure drop is the standard deviation of ten replication experiments. Results showed that the numerical case with less simplification (Case 0 ) compared to experimental data was the case that best predicted the pressure drop for both analyzed velocities, with relative error not exceed 5\%. Case 1 and Case 2 have no significant difference between them, with a higher error of $10 \%$. Figure 3 indicated that Case 3 was the case with the poorest prediction, where the error almost reaches $20 \%$. a smaller velocity peak in the cyclone axis. In the same way, tangential velocity in Figure $4 \mathrm{~b}$ and Figure $4 \mathrm{~d}$ have $\mathrm{a}$ discrepancy in the central position. Case 1 presents higher rotation velocity in a region more width than the real case;

Figure 4 showed the axial and tangential velocity for all numerical cases compared to experimental data to inlet velocities of 10.5 and 12.25 $\mathrm{m} / \mathrm{s}$. The errors bar visualized in Figure 4 represent the standard deviation of three replications of the PIV experiments. All velocity profiles were obtained in the cyclone cylindrical body region, located 40 millimeters below the vortex finder in a horizontal line shape, as present in the left top of Figure 4.

Balestrin et al. (2017) ${ }^{[10]}$ concludes that the inlet ducts used in the experimental results have no influence over the swirling flow and that RSM turbulence model represents the experiments well. Therefore, numerical velocity profile results illustrated in Figure 4 present the effects of the:

- duct after the reduction section-Comparison between Case 0 and Case 1 present that curves and duct after reduction section bring consequences to the central region of the cyclone. Axial velocity profile Figure $4 a$ and Figure $4 c$ present reduction section - Comparing Case 1 and Case 2 was observed that the reduction section present different information of velocities fields in the central location when this apparatus is simulated. The same conclusion obtained with the duct after the reduction can be applied here. Highlighting the less magnitude in the axial peak and the higher width tangential velocity;

- $\quad$ extension of the outlet duct - Comparison between Case 2 and Case 3 shows that the length of the outlet gas tube has a large influence on axial velocity, as seen in Figure $4 a$ and Figure 4c. This discrepancy can be noted because the extension tube shifts the outlet boundary condition far from the cyclone flow, which reduces the downflow probably 
caused by the reverse flows in the outlet boundary. On the other hand, tangential velocity shows a drop in the velocities peak and practically the same velocity in the central region, seen in Figure $4 \mathrm{~b}$ and Figure $4 \mathrm{~d}$.

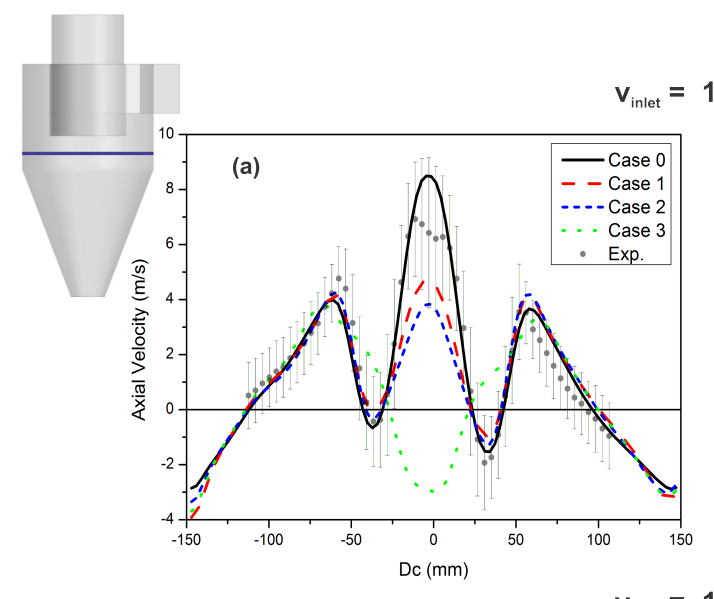

$10.5 \mathrm{~m} / \mathrm{s}$
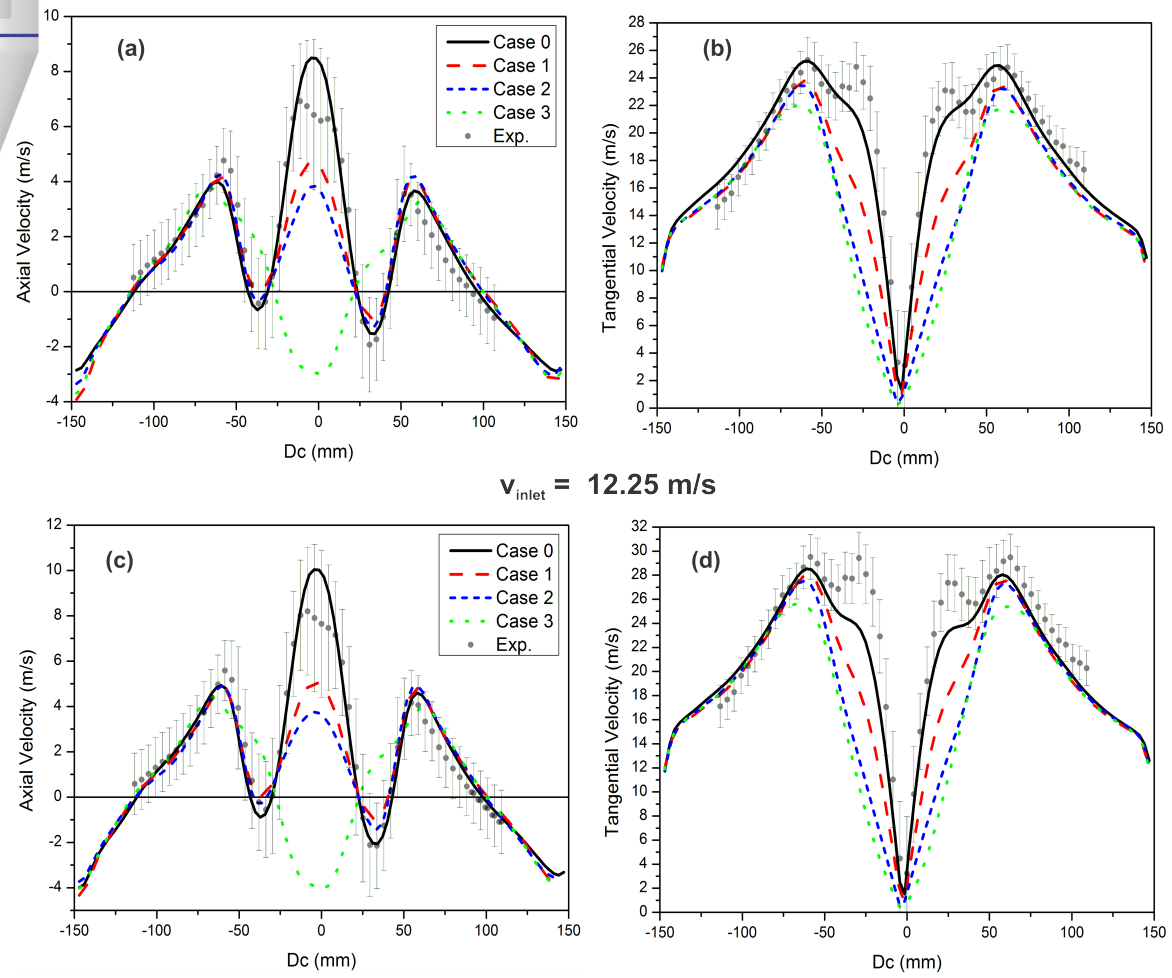

Figure 4: Velocity profiles at inlet velocity $10.5 \mathrm{~m} / \mathrm{s}$ and $12.25 \mathrm{~m} / \mathrm{s}$; (a) (c) axial velocity, (b) (d) tangential velocity.

The effect of boundary conditions that possibly cause the downflow in Case 3, can affect Case 1 and Case 2 as well, but with less loss of information. These changes in the inner cyclone flow influence the pressure drop as shown in Figure 3. By recalling Balestrin et al. ${ }^{[10]}$ discussion, which concludes that the reduction in the transversal section area in the outlet gas duct results in better collection efficiency of particles smaller than $5 \mu \mathrm{m}$, it can be argued that similar consequences in efficiency collection should be expected in the present case.

Therefore, it is possible to conclude that the simplification in the test facility's geometry configuration can have consequences to the cyclone swirling flow. So, the modification in geometries to save computational effort needs caution to ensure catching important issues of the problem under study. The evaluated pressure drop, axial velocity and tangential velocity results showed that Case 0 , which comes closest to the real geometry, is the case that best predicted the experimental data.

\section{Conclusions}

Based on physical experiments and numerical simulation, it was observed that the RSM turbulence model with Reynolds Averaged Navier Stokes equations satisfactorily predicted the pressure drop, axial and tangential velocity in cyclone separators. It was observed differences in trends, the reduction of velocity peaks in the axial and tangential profiles compared to a real case, and the difference of almost $20 \%$ in comparison of Case 0 and Case 3. Therefore, based on these arguments, the authors conclude that simplifications in the test facility's geometric configuration causes modifications in the cyclone swirling flow and so in its performance parameters.

\section{Acknowledgments}

The authors grateful for financial support from Petróleo Brasileiro S.A. PETROBRAS and to Conselho Nacional de Desenvolvimento Científico e Tecnológico - CNPQ, that makes possible this work.

\section{Conflict of interest}

The authors declare that there is no conflict of interest regarding the publication of this manuscript.

\section{Nomenclature}

$r \equiv$ refinement rate;

$q \equiv$ algorithm accuracy formal order;

$\mathrm{F}_{s} \equiv$ safety factor;

$\mathrm{p} \equiv$ pressure $\left[\mathrm{kg} / \mathrm{ms}^{2}\right]$;

$\mathrm{k} \equiv$ turbulent kinetic energy $\left[\mathrm{m}^{2} / \mathrm{s}^{2}\right]$;

$\mathrm{v} \equiv$ velocity $[\mathrm{m} / \mathrm{s}]$;

$\mathrm{g} \equiv$ gravity acceleration $\left[\mathrm{m} / \mathrm{s}^{2}\right]$;

$v \equiv$ molecular kinematic viscosity $\left[\mathrm{m}^{2} / \mathrm{s}\right]$;

$v^{t} \equiv$ turbulence kinematic viscosity $\left[\mathrm{m}^{2} / \mathrm{s}\right]$;

$\mu \equiv$ molecular viscosity $[\mathrm{kg} \mathrm{m} / \mathrm{s}]$;

$\mu^{t} \equiv$ turbulence viscosity $[\mathrm{kg} \mathrm{m} / \mathrm{s}]$;

$\rho \equiv$ density $\left[\mathrm{kg} / \mathrm{m}^{3}\right]$;

$\varepsilon \equiv$ dissipation rate $\left[\mathrm{m}^{2} / \mathrm{s}^{3}\right]$;

$\mathrm{T}^{e} \equiv$ effective tensor $\left[\mathrm{kg} / \mathrm{ms}^{2}\right]$;

$\mathrm{T}^{t} \equiv$ turbulent Reynolds tensor $\left[\mathrm{kg} / \mathrm{ms}^{2}\right]$;

$\mathrm{T} \equiv$ molecular tensor $\left[\mathrm{kg} / \mathrm{ms}^{2}\right]$;

$\mathrm{D}_{\mathrm{j}} \equiv$ turbulent diffusion $\left[\mathrm{kg} / \mathrm{ms}^{3}\right]$;

$\mathrm{D}_{\mathrm{N}} \equiv$ molecular diffusion $\left[\mathrm{kg} / \mathrm{ms}^{3}\right]$;

$\Upsilon \equiv$ production rate $\left[\mathrm{kg} / \mathrm{ms}^{3}\right]$;

$\Pi \equiv$ deformation rate due the pressure $\left[\mathrm{kg} / \mathrm{ms}^{3}\right]$. 


\section{References}

[1] Zenz, Frederick A. (2001). Cyclone-Design Tips, Chemical Engineering, 108 (1).

[2] Nnabalu R. Chinwendu, Falcone Gioia, Bortone Imma. (2019). The Role of Fluid Catalytic Cracking in Process Optimisation for Petroleum Refineries, International Journal of Chemical and Molecular Engineering, 13 (7), 370-376.

https://eprints.gla.ac.uk/190463/7/190463.pdf

[3] Raoufi A., Shams M., Farzaneh M., Ebrahimi R., (2008). Numerical Simulation and Optimization of Fluid Flow in Cyclone Vortex Finder, Chemical Engineering and Processing, 47, 128-137.

https://www.sciencedirect.com/science/article/abs/pii/S025527010700 2607

[4] Papoulias D., Lo S., (2015). Advances in CFD Modelling of Multiphase Flows in Cyclone Separators, Chemical Engineering Transaction, 43, 1603-1608.

https://www.cetjournal.it/index.php/cet/article/view/CET1543268

[5] Hsu C. W., Huang S. H., Lin C. W., Hsiao T. C., Lin W. Y., Chen C. C., (2014). An Experimental Study on Performance Improvement of the Stairmaind Cyclone Design, Aerosol and Air Quality Res., 14, 10031016.

[6] Elsayed K., (2011). Analysis and Optimization of Cyclone Separators Geometry Using RANS and LES Methodologies, PhD Thesis, Vrije Universiteit Brussel, Brussels.

[7] Jo Y., Tien C., Ray M. B., (2000). Development of post cyclone to improve the efficiency of reverse flow cyclones, Powder Technology, 113, 97-108.

https://www.sciencedirect.com/science/article/abs/pii/S003259100000 2060
[8] Chuah T. G., Gimbun J., Choong T. S. Y., (2006). A CFD study of the effect of cone dimensions on sampling aerocyclones performance and hydrodynamics, Powder Technology, 162, 126-132.

https://www.sciencedirect.com/science/article/abs/pii/S003259100500 5498

[9] Souza F. J., Salvo R. V., Martins D. M., (2015). Effects of gas outlet duct length and shape on the performance of cyclone separators, Separation and Purification Technology, 142, 90-100.

https://www.sciencedirect.com/science/article/abs/pii/S138358661400 7539

[10] Balestrin E., Decker R. K., Noriler D., Bastos J. C. S. C., Meier H. F., (2017). An alternative for the collection of small particles in cyclones: Experimental analysis and CFD modeling, Separation and Purification Technology, 184, 54-65. https://www.sciencedirect.com/science/article/abs/pii/S138358661 6323024

[11] Vatankhah H., Zamindar N., Shahedi M., (2016). Geometry Simplification of Wrinkled Wall Semi-rigid Aluminum Containers in Heat Transfer Simulation, Journal of Agricultural Science and Technology, 18, 123-13.

https://www.semanticscholar.org/paper/Geometry-Simplification-ofWrinkled-Wall-Semi-rigid-VatankhahZamindar/44218db621fb89a577581147b7c86175a090ba8c

[12] Taghinia H. J., Rahman M. M., Lu X., (2018). Effects of different CFD modelling approaches and simplification of shape on prediction of flow field around manikin, Energy \& Buildings, 170, 47-60.

https://www.sciencedirect.com/science/article/abs/pii/S037877881733 5648

[13] Schmidt S., Blackburn H. M., Rudman M., (2004). Impact of Outlet Boundary 